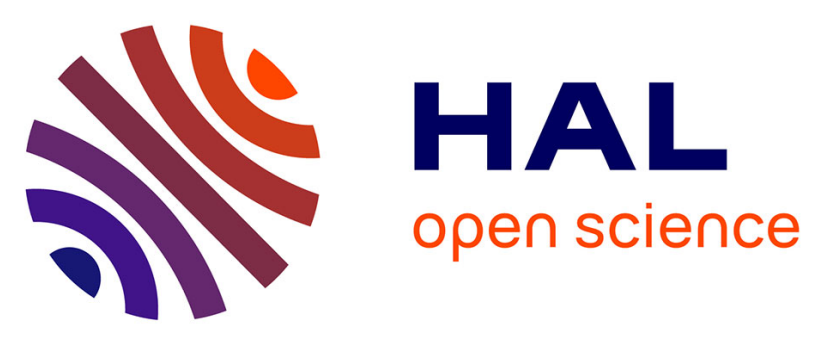

\title{
Antenna Radiation Pattern Measurement in a Reverberating Enclosure Using the Time-Gating Technique
}

Ayoub Soltane, Guillaume Andrieu, Emmanuel Perrin, Cyril Decroze, Alain Reineix

\section{To cite this version:}

Ayoub Soltane, Guillaume Andrieu, Emmanuel Perrin, Cyril Decroze, Alain Reineix. Antenna Radiation Pattern Measurement in a Reverberating Enclosure Using the Time-Gating Technique. IEEE Antennas and Wireless Propagation Letters, 2020, 19 (1), pp.183 - 187. 10.1109/LAWP.2019.2957428. hal-02396456

\section{HAL Id: hal-02396456 \\ https://hal-unilim.archives-ouvertes.fr/hal-02396456}

Submitted on 26 Nov 2020

HAL is a multi-disciplinary open access archive for the deposit and dissemination of scientific research documents, whether they are published or not. The documents may come from teaching and research institutions in France or abroad, or from public or private research centers.
L'archive ouverte pluridisciplinaire HAL, est destinée au dépôt et à la diffusion de documents scientifiques de niveau recherche, publiés ou non, émanant des établissements d'enseignement et de recherche français ou étrangers, des laboratoires publics ou privés. 


\title{
Antenna Radiation Pattern Measurement in a Reverberating Enclosure Using the Time-Gating Technique
}

\author{
Ayoub Soltane, Guillaume Andrieu, Senior Member, IEEE, Emmanuel Perrin, Cyril Decroze and A. Reineix
}

\begin{abstract}
This letter describes an original approach to measure the radiation pattern of an antenna within reverberating enclosures. In such kind of enclosures creating a multipath environment, the key point is to distinguish the line-of-sight (LOS) path between the antenna under test and the measuring antenna from all the other indirect paths. This is made possible by taking advantage of the time-gating technique considering that the LOS path arrives the first on the measuring antenna. With respect to the existing methods proposed recently, the method leads to significantly simplify the measurement setup and shorten the measurement time (similar to the one obtained in anechoic chamber) as no stirring process or any movement of the antenna under test (apart of course from the rotation on itself) is required. Moreover, the accuracy of this method is presumably better in reason of the ability of the time-gating technique to discard the influence of the indirect paths. The proposed technique tested for the first time within reverberating enclosures is experimentally validated on a broadband horn antenna from 1.5 to $12 \mathrm{GHz}$. The main parameters influencing the result (the measurement bandwidth, the frequency resolution, the time-gating window width and the effect of loading the enclosure) are discussed.
\end{abstract}

Index Terms-Anechoic chamber, antenna directivity, lineof-sight, radiation pattern, reverberating enclosure, scattering parameters, stirred and unstirred paths, time-gating.

\section{INTRODUCTION}

$\mathbf{T}$ RADITIONALLY done in anechoic chamber, an increasing interest appears recently in order to characterize the radiation pattern of antennas within reverberating (also called oversized with respect to the wavelength) enclosures. This measurement which may appear counter-intuitive to perform in such a highly multipath environment presents some non negligible advantages. Indeed, such metallic enclosures are not disturbed by the external electromagnetic environment while being substantially less expensive than an anechoic chamber, especially because of the absence of any absorber. Moreover, the efficiency of absorbers used in anechoic chambers is not constant with the frequency and may be insufficient in the lowest frequency range, i.e. below a few hundred of $\mathrm{MHz}$ depending on the size of the absorbers.

In a multipath environment, the challenge for radiation pattern measurement is to measure the line-of-sight (LOS)

Manuscript received October 23, 2019, revised November 25, 2019, accepted December 1, 2019. This work is supported by the XLIM laboratory. (Corresponding author: Guillaume Andrieu.)

A. Soltane, G. Andrieu, C. Decroze and A. Reineix are with the Xlim laboratory, 123 avenue Albert Thomas, 87000 Limoges, France (e-mail: ayoub. soltane, guillaume.andrieu, cyril.decroze and alain.reineix@xlim.fr).

E. Perrin is with CISTEME, 12 rue Gémini, Bâtiment 2, 87068 Limoges, France (e-mail: perrin@cisteme.net). path between both antennas while discarding the influence of all the other indirect or non-LOS (NLOS) paths reflected by the enclosure walls. To our knowledge, three methods described hereafter have already been proposed to perform such measurements in reverberating enclosures (RE).

A first method [1] based on the determination of the $K$ factor [2]- [3] in a mechanically stirred reverberation chamber (RC) (a RC being an RE equipped of a mode stirring process) has been proposed. The $K$-factor being the ratio of the power related to the unstirred and to the stirred paths within the enclosure, this allows the influence of the stirred ones to be discarded. However, this method suffers of the impossibility to distinguish LOS and unstirred NLOS paths having no interaction with the mode stirrer. Therefore, the method offers a weak accuracy and is limited to the angles related to the main lobes of the antenna. Moreover, the accurate determination of the RC $K$-factor for each AUT angle involves a long measurement time.

The Doppler effect produced by the rectilinear motion (stepwise [4] or in continuous motion [5]) of the AUT has also been proposed. The knowledge of the velocity (which has to be constant all along the displacement of the AUT) allows the Doppler shift frequency related to the LOS signal (in reality the maximum Doppler shift of the Doppler spectrum) to be predicted [6]. The amplitude of the Doppler spectrum at this particular frequency for each AUT angle leads to the antenna radiation pattern. As the method accuracy is directly related to the AUT displacement, this method requires a long motorized rail and involves a long measurement time.

Finally, a third method [7] based on a spherical wave decomposition has been proposed recently in an RC. The aim is to measure the $S_{21}$ scattering parameter between two antennas for two different positions of the AUT, each time for a large number of stirring conditions. The computation of self-correlation coefficients between both positions of the AUT allows the radiation pattern of the AUT to be reconstructed.

All these methods involve a long measurement time and a large complexity of the experimental setup (a mode stirring process or at least a rectilinear displacement of the AUT being required). In addition, the accuracy of these methods is limited mainly in reason of the difficulty to distinguish the LOS and the first unstirred NLOS paths.

Already validated for radiation pattern measurements in imperfect anechoic environments [8]- [9] as for instance in outdoor environments in order to remove the effect of ground reflections, the time-gating method is tested for the same 
application for the first time in REs. It is demonstrated in this letter that this technique outmatches the existing methods in such multipath environment in terms of simplicity of the measurement setup and duration of the measurements. The measurement accuracy is also presumably better as the timegating technique is able to perfectly cancel the influence of the indirect paths.

\section{Description Of The Time-GAting Method}

The measurement principle consists in measuring the LOS signal traveling the distance $d_{L O S}$ (i.e. arriving at the instant $t_{L O S}$ ) between the AUT and the measuring antenna. The aim is to discard all the NLOS paths and especially the one traveling the shortest distance $d_{N L O S}^{\min }$ (arriving at $t_{N L O S}^{\min }$ ).

This is achieved by measuring the scattering parameter $S_{21}$ between both antennas located at the same height for any couple of angles $(\theta, \phi)$. Each measurement is performed on a bandwidth $B$ centered around the frequency of interest $f_{0}$ (with $B<f_{0}$ ).

Then, the impulse response of the system made of both antennas is computed with an inverse Fourier transform (IFT) of $S_{21}(f, \theta, \varphi)$

$$
h(t, \theta, \varphi)=I F T\left[S_{21}(f, \theta, \varphi) . W(f)\right]
$$

with $W(f)$ the frequency domain windowing function. The rectangular and the Hann filter have been tested in this letter, the latter being able to reduce the influence of the secondary lobes (but also to enlarge the main lobe) of the impulsional response with respect to the rectangular window.

The time-gating technique consists in truncating the signal at a time $t_{t g}$ included in the range between $t_{L O S}$ and $t_{N L O S}^{m i n}$, i.e. after the arrival of the LOS path on the measuring antenna but before the arrival of all the other paths. More exactly, we realize a zero-padding operation on all the points not included in the time-gating window in order to keep the same frequency domain resolution at the end of the post-treatment process.

The final step consists in doing the Fourier transform of the truncated signal and then examine its value at $f_{0}$ (or in theory at any frequency included in the bandwidth $B$ ). The radiation pattern (in 2 or 3 dimensions) is obtained after repeating this process for each desired couple of angles $(\theta, \varphi)$ at a given frequency.

The measuring antenna has to be located in the far-field of the AUT. Respecting this condition, the distance $d_{L O S}$ has to be chosen in order to increase (if possible) the relative difference between $d_{L O S}$ and $d_{N L O S}^{\min }$. This can help to measure the radiation pattern of dispersive or resonating antennas. In such case, the measurement is possible if the spread signal related to the LOS path is entirely received on the measuring antenna before the arrival of the first NLOS path.

Different measurement parameters influence the results obtained by this approach. First, the choice of the bandwidth $B$ is fundamental in order to dissociate the LOS and the shortest NLOS path. The minimum bandwidth $B_{\min }$ of the $S_{21}$ measurement equals $c / \delta_{d}^{\max }$ with $c$ the speed of light and $\delta_{d}^{\max }$ the maximum tolerable spatial resolution related to the difference of distance between $d_{L O S}$ and $d_{N L O S}^{\min }$

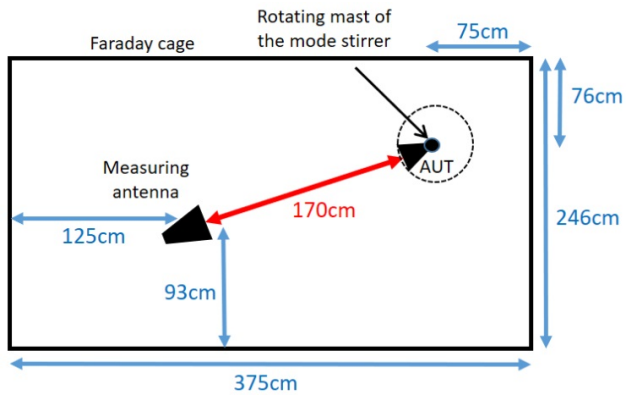

Fig. 1. Geometrical description (top view) of the experimental setup used in the RE of the XLIM laboratory. According to the distance bewteen both antennas, the LOS should theoretically arrive at $t=5.5 \mathrm{~ns}$ when neglecting the additional propagation time related to both antennas themselves.

$$
B_{\min }=c /\left(d_{N L O S}^{\min }-d_{L O S}\right)=1 /\left(t_{N L O S}^{\min }-t_{L O S}\right)
$$

Second, the frequency domain resolution $\delta_{f}$ of the $S_{21}$ measurement is also important in order to avoid any aliasing effect when performing the inverse Fourier transform. Indeed, a $\delta_{f}$ parameter insufficiently small has the effect to increase the results of the inverse Fourier transform in all the time domain and especially during the first instants. This adds a non-physical noise reducing the signal to noise ratio within the time-gating window. The last parameters influencing the results are the frequency domain windowing function and the fact to insert absorbers within the enclosure [10]- [11]. The influence of all these parameters is discussed in the validation section of this letter.

\section{VALIDATION}

The validation of the proposed method has been made on a double ridge horn antenna (i.e., the AUT) working in the frequency range from 0.75 to $18 \mathrm{GHz}$, the measuring antenna being an antenna of the same model pointing towards the AUT. Reference results have been obtained in one anechoic chamber of the XLIM laboratory where both antennas were located at a distance from each other around $7 \mathrm{~m}$. Measurements have been performed on both E- and H-planes from 1.5 to $12 \mathrm{GHz}$ over 22 frequencies (frequency step of $0.5 \mathrm{GHz}$ ), each with an angular step of $1^{\circ}$ (for the sake of brevity, H-plane results are not shown in this letter but the conclusions are the same).

The experimental setup used in the RC of the XLIM laboratory $(3.57 \mathrm{~m}$ long, $2.45 \mathrm{~m}$ wide, $2.46 \mathrm{~m}$ high, volume $V \approx 21.5 \mathrm{~m}^{3}$, theoretical fundamental resonance $f_{\text {fund }} \approx 74 \mathrm{MHz}$ ) is described in Figs. 1-2. The rotating mode stirrer being removed (the enclosure being no longer an $\mathrm{RC}$ but an RE), the AUT has been fixed to its rotating mast. $S_{21}$ measurements have been performed on the bandwidth from 1 to $13 \mathrm{GHz}$ over 48001 points corresponding to a frequency shift $\delta_{f}$ of $0.25 \mathrm{MHz}$ between two successive frequencies. The measurements have been repeated for the RE left empty (i.e. the "unloaded" configuration) and when three blocks of absorbers have been inserted in (i.e. the "loaded" configuration).

Fig. 3 presents the power-delay profile [12]- [13] (averaged over all the angles) obtained in the RE for both loading 


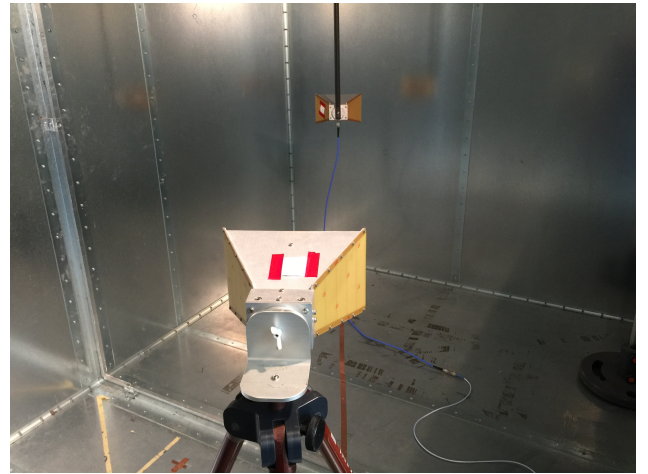

Fig. 2. Picture of the experimental setup used in the RE of the XLIM laboratory. The measurement antenna is in the foreground while the AUT is in the background. Orientation of antennas correspond to a H-plane radiation pattern measurement (here for the angle $\theta=-180^{\circ}$ ). The influence of the movement of the blue coaxial cable connected to the AUT during its rotation is assumed to be negligible in our results.

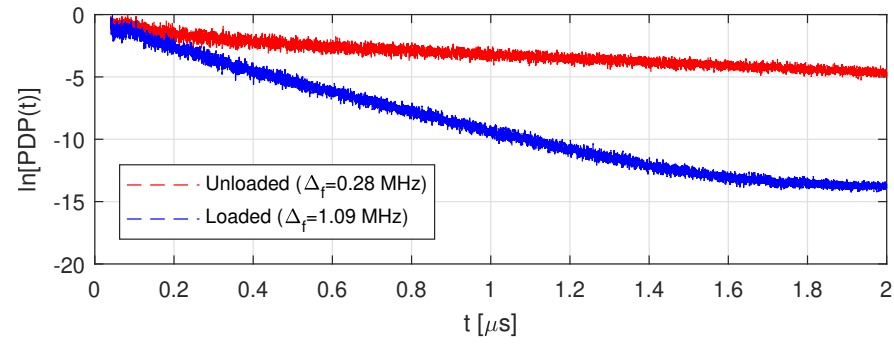

Fig. 3. Normalized power delay profile of the RE for both loading configurations.

configurations as well as the average mode bandwidth $\Delta_{f}$ defined as the ratio between the frequency $f$ and the average composite quality factor $Q$ [14]- [15].

For each particular configuration, the measurements last around 260 minutes. This long measurement time can not be directly compared to the measurement time in anechoic chamber. Indeed, in such measurements, it is possible to compute the radiation pattern using the time-gating technique on the whole frequency range (with the exception of the bounds) and not only at 22 particular frequencies. Moreover, it should be noted than 60 minutes are devoted to the stabilization of the AUT after each movement (i.e. 10 seconds for each angle).

Before applying the time-gating process on these results, we have first identified the instant of arrival of the LOS and of the first three NLOS paths in order to determine a suitable value of $t_{t g}$. To do this, we have computed the absolute value of the IFT of $S_{21}$ for each angle and then taken the maximum value obtained at any instant. It is shown in Fig. 4 that these paths arrives at respectively $t=7.5 \mathrm{~ns}$ for the LOS and $t=10.8$, 12.3 and $14.5 \mathrm{~ns}$ for the first three NLOS paths. Therefore, $t_{t g}$ has to be included in the range between 7.5 and $10.8 \mathrm{~ns}$ and the minimum bandwidth $B$ equals $300 \mathrm{MHz}$ using (2).

For each sub-band $B=1 \mathrm{GHz}$ around each frequency where the radiation pattern has been measured in $\mathrm{AC}$, we have computed the truncated signal (using a symmetrical timegating window from $-t_{t g}$ to $t_{t g}$ ) for each angle of the radiation pattern (as shown in Fig. 5 for two particular angles) and then the radiation pattern of the AUT. The excellent agreement

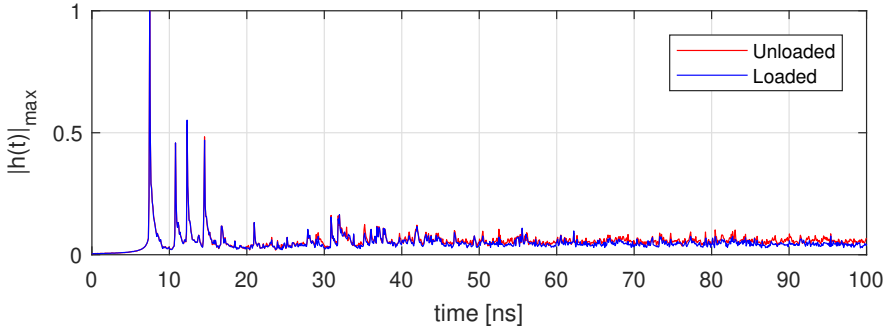

Fig. 4. Normalized maximum impulsional response $|h(t)|_{\max }$ in the E-plane from $S_{21}$ measurements performed from 1 to $13 \mathrm{GHz}$ over 48001 frequency points for both loading configurations of the RE. The LOS and the first three NLOS paths appears clearly. It is also shown that the insertion of 3 blocks of absorbers does not have any influence during the early instants but becomes gradually visible after.

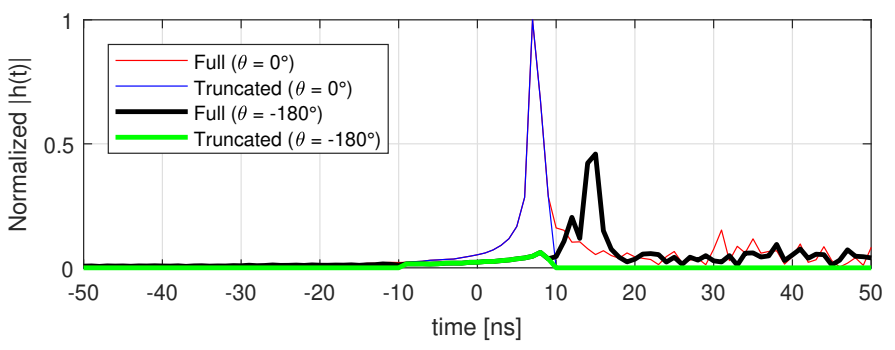

Fig. 5. Full and truncated (with $t_{t g}=9 \mathrm{~ns}$ ) magnitude of the impulsional response $|h(t)|$ around $f=12 \mathrm{GHz}$ (with $B=1 \mathrm{GHz}$ ) in the E-plane for two different $\theta$ angles. These curves are normalized with respect to the $\theta=0^{\circ}$ plot. It appears clearly that the LOS has a lower amplitude with respect to the first two NLOS paths for $\theta=-180^{\circ}$.
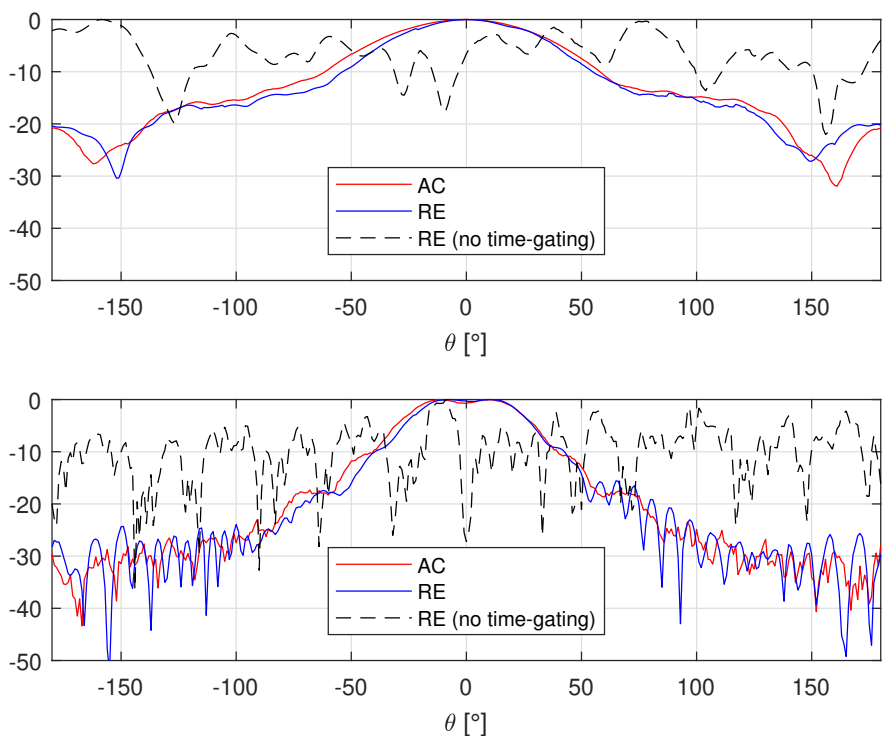

Fig. 6. Normalized radiation pattern (in $\mathrm{dB}$ ) in the E-plane at 1.5 and $12 \mathrm{GHz}$ in the anechoic chamber and the unloaded RE in the optimal conditions for time-gating ( $B=1 \mathrm{GHz}, \delta_{f}=0.25 \mathrm{MHz}$, symmetric window). Results obtained without any post-treatment are as expected totally erroneous.

obtained in Fig. 6 validates the time-gating procedure method in RE.

In order to quantify on a rigorous basis the differences between the reference radiation pattern $D_{A C}$ measured in the anechoic chamber and the one $D_{R E}$ measured in our RE, we have computed the root-mean-square error (RMSE) as follows 
TABLE I

Average RMSE (IN DB) IN THE E-PLANE FOR $\delta_{f}=250 \mathrm{KHZ}$ AND A SYMMETRIC TIME-GATING WINDOW

\begin{tabular}{|c|c|c|c|c|}
\hline$B$ [GHz] & 0.25 & 0.5 & 0.75 & 1 \\
\hline time-domain resolution $\delta_{t}[\mathrm{~ns}]$ & 4 & 2 & 1.33 & 1 \\
\hline$t_{t g}[\mathrm{~ns}]$ & 8 & 8 & 9.33 & 9 \\
\hline Unloaded RE \& Unfiltered & -26.5 & -27.8 & -29.8 & -29.7 \\
\hline Unloaded RE \& Hann-filtered & -23 & -28 & -28.1 & -29.8 \\
\hline Loaded RE \& Unfiltered & -26.8 & -28.1 & -30.1 & -30.2 \\
\hline Loaded RE \& Hann-filtered & -23 & -28.3 & -28.5 & -30.3 \\
\hline
\end{tabular}

TABLE II

AVERAGE RMSE (IN DB) IN THE E-PLANE FOR $\delta_{f}=3$ MHZ AND A SYMMETRIC TIME-GATING WINDOW

\begin{tabular}{|c|c|c|c|c|}
\hline$B[\mathrm{GHz}]$ & 0.25 & 0.5 & 0.75 & 1 \\
\hline$t_{t g}[\mathrm{~ns}]$ & 10 & 9 & 9.33 & 9 \\
\hline Unloaded RE \& Unfiltered & -17.3 & -18.6 & -17.2 & -19.2 \\
\hline Unloaded RE \& Hann-filtered & -14.9 & -16.8 & -17.9 & -17.8 \\
\hline Loaded RE \& Unfiltered & -26.3 & -27.2 & -28.1 & -26.9 \\
\hline Loaded RE \& Hann-filtered & -23.2 & -27.2 & -27.5 & -26.5 \\
\hline
\end{tabular}

$$
R M S E=\sqrt{\left\langle\left[D_{A C}(\alpha)-D_{R E}(\alpha)\right]^{2}\right\rangle}
$$

for each entire radiation pattern, $\alpha$ being the angle $\theta$ for the E-plane and $\phi$ for the H-plane. This error computed for one particular frequency has been averaged over the 22 considered frequencies and converted in $\mathrm{dB}$ with $\left\langle R M S E_{d B}\right\rangle=$ $20 \log _{10}(\langle R M S E(f)\rangle)$.

It is worth noting here that measurements performed in both facilities (and therefore also in AC) are subject to measurement uncertainties (in particular positioning errors) especially near the nulls of the beam pattern.

The average RMSE presented in Table I for four different bandwidth $B$ (including a value of $0.25 \mathrm{GHz}$ lower than $B_{\text {min }}$ ) has been calculated for both loading configurations with and without a Hann filtering. An average RMSE lower than $-28 \mathrm{~dB}$ for $B>0.75 \mathrm{GHz}$ is obtained which can be considered as really satisfying. The decrease of accuracy for lower $B$ values appears clearly. On the contrary, the benefit to use a Hann filter or to load the RE is not clearly visible here.

To reduce the measurement time, it can be of interest to decrease the number of collected frequencies during the $S_{21}$ measurements. We have therefore repeated the measurement in the RC at only 4001 frequencies on the same frequency range (i.e. $\delta_{f}=3 \mathrm{MHz}$ ). The total measurement time reduces to 83 minutes (with one hour still related to the stabilization of the AUT). Table II containing the results obtained for the same configurations from this new measurement conditions clearly illustrates the degradation of the results quality, particularly in the case of the "unloaded" RE, the results obtained for the loaded configuration remaining satisfying. These results illustrates the importance of the aliasing effect in case of an insufficient frequency domain resolution. Indeed, even if the time-gating truncates the signal early, it is fundamental to have a sufficiently high $t_{\max }$ related to the IFT, which is obtained with a sufficiently low $\delta_{f}$. Otherwise, aliasing effects have the effect to add a non-physical offset in the early time of the impulsional response. This is illustrated in Fig. 7 where the

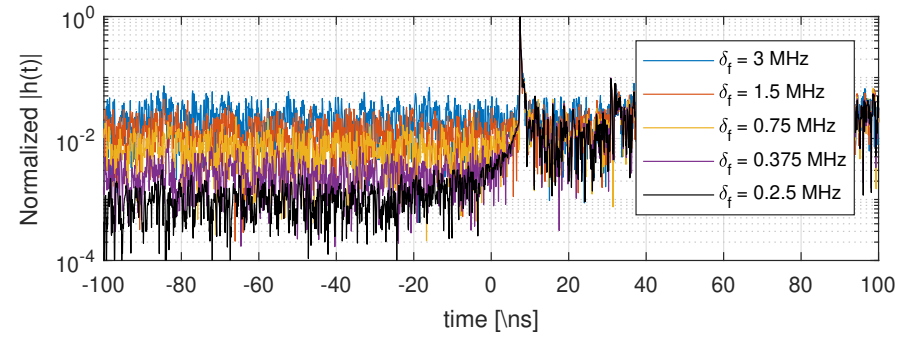

Fig. 7. Aliasing effect as a function of the frequency domain resolution.

TABLE III

Average RMSE (IN DB) IN THE E-PLANE FOR $\delta_{f}=3$ MHZ AND A "TIGHT" TIME-GATING WINDOW

\begin{tabular}{|c|c|c|c|c|}
\hline$B[\mathrm{GHz}]$ & 0.25 & 0.5 & 0.75 & 1 \\
\hline$t_{t g 1}[\mathrm{~ns}]$ & 6 & 7 & 6.66 & 7.5 \\
\hline$t_{t g 2}[\mathrm{~ns}]$ & 10 & 9 & 8 & 8.5 \\
\hline$N$ & 2 & 2 & 2 & 2 \\
\hline Unloaded RE \& Unfiltered & -21.6 & -24.4 & -25.8 & -26.4 \\
\hline Unloaded RE \& Hann-filtered & -19 & -23.8 & -25.2 & -26.1 \\
\hline Loaded RE \& Unfiltered & -27.5 & -27.2 & -26.8 & -26.5 \\
\hline Loaded RE \& Hann-filtered & -23.5 & -28.2 & -28.3 & -28.1 \\
\hline
\end{tabular}

normalized $h(t)$ is shown for different number of frequencies collected from 1 to $13 \mathrm{GHz}$. It appears clearly that the aliasing increases artificially the level of $h(t)$ in the early instants even if the peak related to the LOS at $t=7.5 \mathrm{~ns}$ seems not to be disturbed. This phenomenon also explains why loading the chamber improves the results. Indeed, the loading accelerates the decrease of energy which reduces the aliasing during the early instants.

In addition with the potential insertion of absorbers which can solve the problem, another solution consists in reducing the time-gating window around the peak related to the LOS path in order to cancel the unwanted signal around it. Table III contains the average RMSE in the case of a "tight" timegating window (from $t_{t g 1}$ to $t_{t g 2}, N$ being the number of points included in the time-gating window) for $\delta_{f}=3 \mathrm{MHz}$. These results show an enhancement of the time-gating performances for both loading configurations. However, there is still some differences between the unloaded and the loaded configurations as the aliasing effect still disturbs the LOS peak especially for the angles when the radiation pattern is small.

\section{CONCLUSION}

We have presented in this letter an application of the timegating technique in order to measure the radiation pattern of antennas in oversized RE (and not necessarily RCs). The method is applicable on broadband antennas, i.e. sufficiently matched on the measurement bandwidth $B\left(\left|S_{11}\right|<-10 \mathrm{~dB}\right)$, and of any directivity.

With respect to the state-of-the-art in REs or RCs, the method involves a simpler experimental setup (no mode stirring or AUT displacement being required except the rotation on itself) and a measurement time almost identical to the one obtained in anechoic chamber. A better accuracy is also expected in reason of a better removal of the indirect NLOS paths. The influences of all the measurement parameters influencing the results have been discussed in this letter. 


\section{REFERENCES}

[1] C. Lemoine, E. Amador, P. Besnier, J. Floc'h, and A. Laisné, "Antenna directivity measurement in reverberation chamber from rician k-factor estimation," IEEE Transactions on Antennas and Propagation, vol. 61, no. 10 , pp. 5307-5310, Oct 2013.

[2] C. Lemoine, E. Amador, and P. Besnier, "On the $k$-factor estimation for rician channel simulated in reverberation chamber," IEEE Transactions on Antennas and Propagation, vol. 59, no. 3, pp. 1003-1012, March 2011.

[3] C. L. Holloway, D. A. Hill, J. M. Ladbury, P. F. Wilson, G. Koepke, and J. Coder, "On the use of reverberation chambers to simulate a rician radio environment for the testing of wireless devices," IEEE Transactions on Antennas and Propagation, vol. 54, no. 11, pp. 3167-3177, Nov 2006.

[4] M. A. García-Fernández, D. Carsenat, and C. Decroze, "Antenna radiation pattern measurements in reverberation chamber using plane wave decomposition," IEEE Transactions on Antennas and Propagation, vol. 61, no. 10, pp. 5000-5007, Oct 2013.

[5] _ _. "Antenna gain and radiation pattern measurements in reverberation chamber using doppler effect," IEEE Transactions on Antennas and Propagation, vol. 62, no. 10, pp. 5389-5394, Oct 2014.

[6] A. Soltane, G. Andrieu, and A. Reineix, "Doppler spectrum analysis for the prediction of rotating mode stirrer performances in reverberation chamber," IEEE Transactions on Electromagnetic Compatibility, pp. 16, 2018.

[7] Q. Xu, Y. Huang, L. Xing, C. Song, Z. Tian, S. S. Alja'afreh, and M. Stanley, "3-d antenna radiation pattern reconstruction in a reverberation chamber using spherical wave decomposition," IEEE Transactions on Antennas and Propagation, vol. 65, no. 4, pp. 1728-1739, April 2017.

[8] S. Loredo, M. Pino, F. Las-Heras, and T. Sarkar, "Echo identification and cancellation techniques for antenna measurement in non-anechoic test sites," IEEE Antennas and Propagation Magazine, vol. 46, no. 1, February 2004.

[9] P. Piasecki and J. Strycharz, "Measurement of an omnidirectional antenna pattern in an anechoic chamber and an office room with and without time domain signal processing," in 2015 Signal Processing Symposium (SPSympo), June 2015, pp. 1-4.

[10] A. Cozza, "The role of losses in the definition of the overmoded condition for reverberation chambers and their statistics," IEEE Transactions on Electromagnetic Compatibility, vol. 53, no. 2, pp. 296-307, May 2011.

[11] A. Adardour, G. Andrieu, and A. Reineix, "On the low-frequency optimization of reverberation chambers," IEEE Transactions on Electromagnetic Compatibility, vol. 56, no. 2, pp. 266-275, April 2014

[12] E. Genender, C. L. Holloway, K. A. Remley, J. M. Ladbury, G. Koepke, and H. Garbe, "Simulating the multipath channel with a reverberation chamber: Application to bit error rate measurements," IEEE Transactions on Electromagnetic Compatibility, vol. 52, no. 4, pp. 766-777, Nov 2010.

[13] C. L. Holloway, H. A. Shah, R. J. Pirkl, K. A. Remley, D. A. Hill, and J. Ladbury, "Early time behavior in reverberation chambers and its effect on the relationships between coherence bandwidth, chamber decay time, rms delay spread, and the chamber buildup time," IEEE Transactions on Electromagnetic Compatibility, vol. 54, no. 4, pp. 714-725, Aug 2012.

[14] P. S. Kildal, X. Chen, C. Orlenius, M. Franzen, and C. S. L. Patane, "Characterization of reverberation chambers for ota measurements of wireless devices: Physical formulations of channel matrix and new uncertainty formula," IEEE Transactions on Antennas and Propagation, vol. 60, no. 8, pp. 3875-3891, Aug 2012.

[15] G. Andrieu, N. Ticaud, F. Lescoat, and L. Trougnou, "Fast and accurate assessment of the "well stirred condition" of a reverberation chamber from $s_{11}$ measurements," IEEE Transactions on Electromagnetic Compatibility, vol. 61, no. 4, pp. 974-982, Aug 2019. 